\title{
FRATURA DENTAL COM TRATAMENTO MULTIDISCIPLINAR
}

\section{DENTAL FRACTURE WITH MULTIDISCIPLINARY TREATMENT}

\section{Raisa HINTZ ${ }^{*}$, Fabrício Rutz da SILVA ${ }^{2}$}

${ }^{1}$ Mestre em Dentística, pela Pontifícia Universidade Católica do Paraná. Professora no Centro Universitário de União da Vitória - UNIUV. E-mail: prof.raisa@uniuv.edu.br

${ }^{2 *}$ Autor para contato: Mestre em Odontologia em Saúde Coletiva pela FOP-UNICAMP. Professor no Centro Universitário de União da Vitória - UNIUV. Rua Professor João Falarz, 555, Orleans - Curitiba - Paraná - Brasil. CEP: 81280270 | Telefone: 55 (41) 33081888. E-mail:prof.fabricio@uniuv.edu.br

\section{RESUMO}

Paciente de 11 anos de idade, sexo masculino, fraturou o incisivo central superior direito. A fratura envolveu $2 / 3$ da coroa, comprometimento pulpar, extensão subgengival na face palatina e invasão de espaço biológico. Os procedimentos realizados foram: gengivoplastia, tratamento endodôntico, tracionamento dental e colagem do fragmento associada à colocação de pino intrarradicular. Concluiu-se que a associação de diferentes áreas da odontologia permite resultados satisfatórios para o restabelecimento estético e funcional de fraturas dentais e suas sequelas.

Palavras-chave: Colagem dentária. Pinos dentários. Fraturas dos dentes.

\begin{abstract}
Patient 11 years old, male, fractured maxillary central incisor. The fracture involved $2 / 3$ of the crown, pulp involvement, subgingival extension on the palatal face and biological space invasion. The procedures developed were: gingivoplasty, endodontic treatment, dental pick and fragment bonding associated with the placement of intra root post. The results showed that the combination of different areas of dentistry could provide satisfactory results for the aesthetic and functional of dental fractures and their sequelae.
\end{abstract}

Keywords: Dental bonding. Dental pins. Fractures in teeth. 


\section{INTRODUÇÃO}

As fraturas dentais têm uma incidência de $1 \%$ a $3 \%$ na população mundial (MAZZOLENI et al., 2016). Em crianças, sua prevalência é de $20 \%$ e, em adultos, 30\% (ANDERSSON, 2013). Acidentes automobilísticos e esportivos são suas causas mais comuns, sendo os incisivos superiores os dentes mais acometidos (LEVIN et al., 2007; LO GIUDICE et al., 2012). Entre as várias modalidades de injúrias dentais, as fraturas de dentes permanentes destacam-se em relação aos dentes decíduos (cerca de 75\% dos casos) (ANDREASEN; LAURIDSEN; ANDREASEN, 2010; GONG et al., 2011; ANDERSSON, 2013).

O conhecimento e o diagnóstico das fraturas dento-alveolares, de acordo com sua localização e grau de comprometimento, são fatores primordiais para o estabelecimento de um correto plano de tratamento (GÓES et al., 2005). Os avanços na odontologia adesiva oferecem opções variadas para recolocação de fragmentos fraturados com base na amplitude, na direção e na gravidade da fratura (MANJU et al., 2015).

As fraturas podem ser classificadas em: dento-alveolares, coronárias, coronárias com envolvimento radicular, radiculares, do processo alveolar, concussão, subluxação, extrusão, luxação lateral, intrusão e avulsão (ANDREASSEN et al., 2001). Contudo, o cirurgião-dentista não deve deter-se apenas aos danos envolvendo os tecidos duros, pois as lacerações em tecidos moles podem estar associadas ao traumatismo dento-alveolar (PETERSON et al., 2000).

Quando o profissional se depara com traumatismo dento-alveolar, é de capital importância a realização de uma boa anamnese (Quando? Onde? Como?) associada a exames radiográficos. No entanto, são necessários também testes de mobilidade, de percussão e de sensibilidade; exames estes que contribuem para diagnóstico do comprometimento das estruturas de suporte do elemento traumatizado (GÓES et al., 2005). O tipo de tratamento prestado depende, principalmente, da extensão da fratura, do envolvimento pulpar, da fratura radicular, da violação do espaço biológico e da presença do fragmento fraturado (YOUSEF, 2015).

A colagem direta de telas, de botões e de bráquetes ortodônticos, normalmente usados para tração de dentes inclusos, transformou-se na técnica preferida para o tratamento de recuperação das distâncias biológicas, pois, além de maior facilidade, exige menor extensão cirúrgica e remoção de tecido para acesso à coroa dental do que as outras técnicas (MORAES et al., 2008). Várias técnicas de extrusão dental estão disponíveis, sendo a que utiliza aparelhos fixos normalmente bem tolerada e requer apenas uma mínima cooperação por parte do paciente (DURHAM; GODDARD; MORRISON, 2004).

A utilização de pinos estéticos tem facilitado as restaurações de dentes tratados endodonticamente. Suas propriedades como transmissão de luz, biocompatibilidade, adesão à estrutura dental e ao material de restaurador, módulo de elasticidade próximo do dente, resistência à corrosão e estética fazem com que o pino estético seja o material de primeira escolha para restaurações estéticas (FERRARI; VICCHI, 2000). A condição periodontal e endodôntica, comprimento da raiz e as estruturas histológicas de dentes desvitalizados também devem ser consideradas para o sucesso do tratamento (QUINTAS; DINATO; BOTTINO, 2000).

A evolução permanente da ciência e da tecnologia obriga os profissionais a estarem atualizados e aptos a realizar o melhor tratamento disponível para o caso, baseando sua escolha em evidências científicas (SHARMA et al., 2011).

\section{Caso clínico}

Paciente masculino M.J.T., 11 anos, apresentou-se à clínica odontológica da PUC/PR, relatando fratura dental do elemento 11. Durante o exame clínico, verificou-se que o paciente apresentava fratura no sentido oblíquo de esmalte/dentina, com envolvimento pulpar e infraóssea (Figuras 1 e 1A).

Figura 1 - Aspecto inicial da fratura esmalte dentina

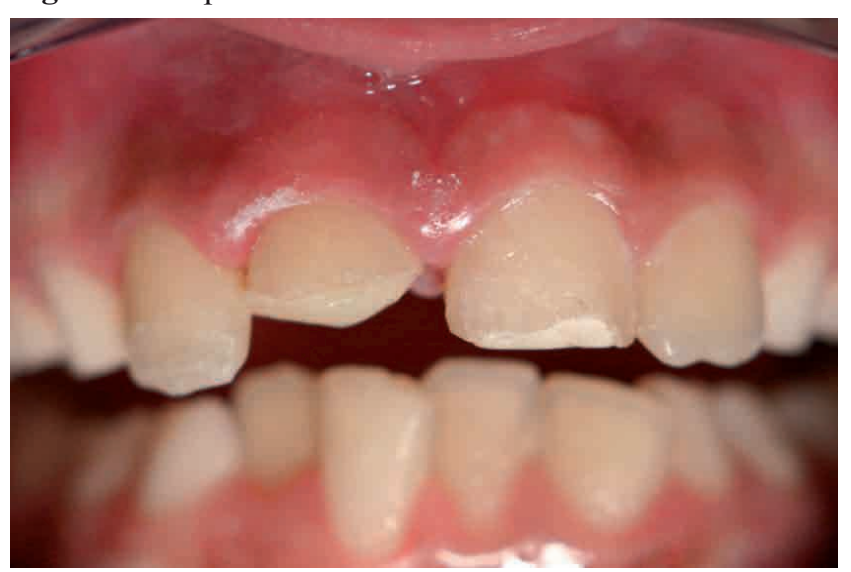


Figura 1 A - Aspecto inicial da fratura

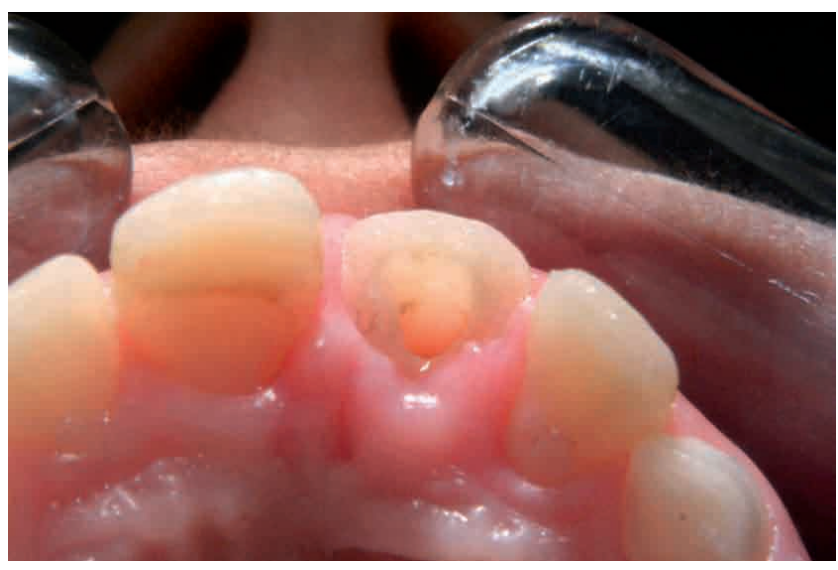

O paciente relatou que o fragmento dental foi colocado em soro fisiológico, solução que ele tinha em casa no momento do acidente, sendo mantido nessa substância até que o atendimento odontológico fosse realizado. Assim, na primeira sessão, foi feita anamnese, exames clínico e radiográfico além da profilaxia de todos os dentes, com pedra pomes e água. Em seguida, foi realizada a técnica cirúrgica de gengivoplastia para remoção do excesso gengival (Figura 1B), visando aumentar a coroa clínica para fixação do grampo do isolamento absoluto e posterior tratamento endodôntico.

Figura 1 B - Gengivoplastia

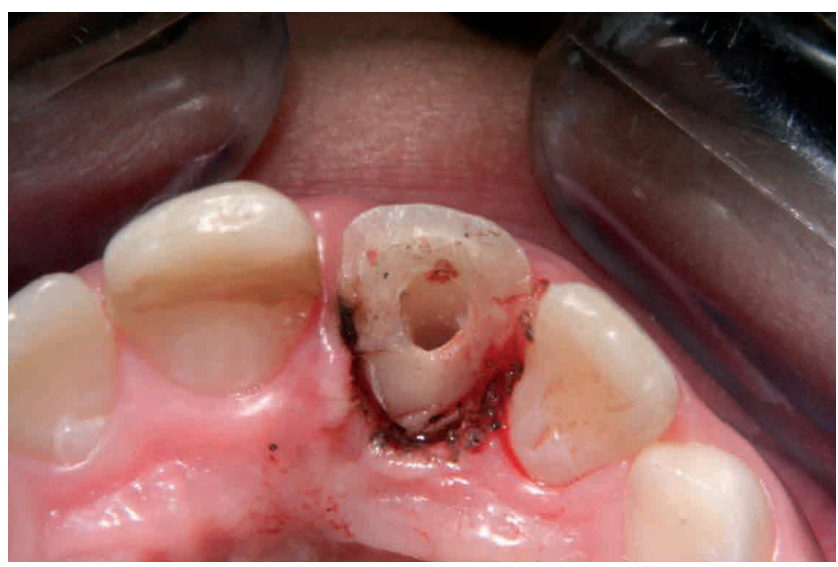

O tratamento endodôntico foi realizado em única sessão, pois este se apresentava com vitalidade pulpar. $\mathrm{O}$ canal foi instrumentado e obturado com cimento Endofil (Dentsply, York, PA, EUA) (Figura 2).
Figura 2 - Radiografia do canal obturado

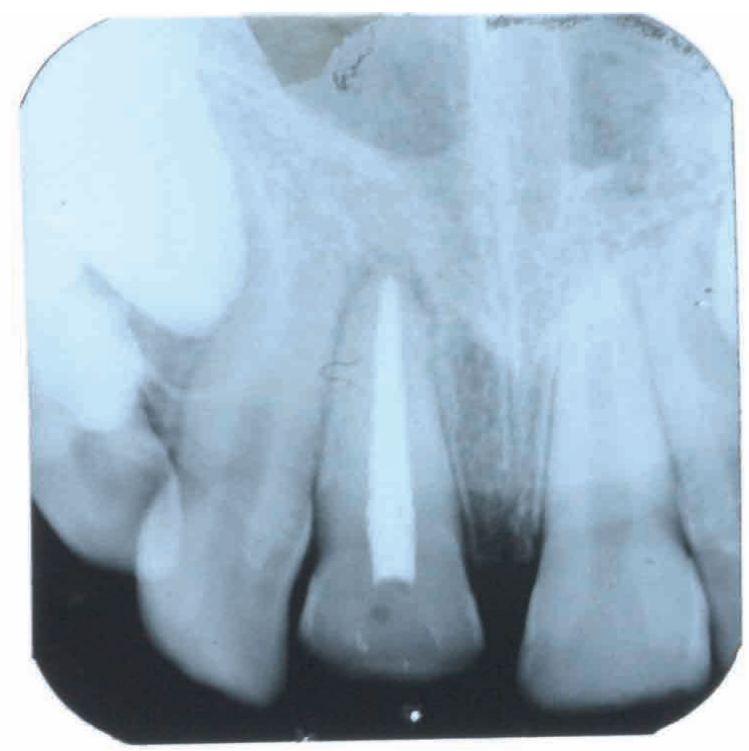

Para não haver comprometimento estético na região anterior, decorrente de uma osteotomia para obtenção dos espaços biológicos, foi optado o tracionamento ortodôntico, pela técnica do tracionamento rápido. Inicialmente, foi realizada a fribrotomia ao redor do dente, com a finalidade de descolar as fibras do ligamento periodontal, auxiliando, assim, na extrusão dental. O tracionamento foi realizado com a inserção de um fio ortodôntico em forma de gancho no interior do conduto radicular cimentado com cimento fosfato de zinco (SS White, Rio de Janeiro, Brasil) (Figura 3).

Figura 3 - Fio ortodôntico no interior do conduto

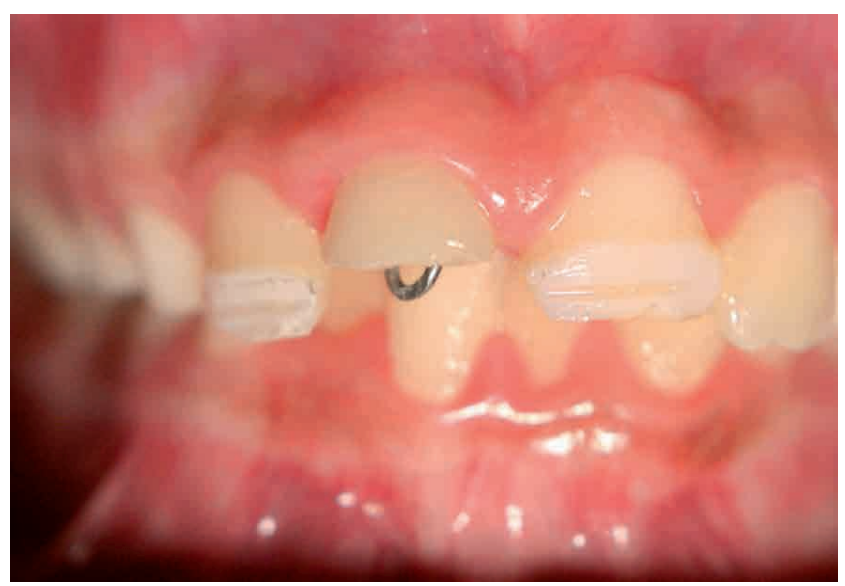

Nos dentes vizinhos, foi realizado o condicionamento ácido e utilizado o sistema adesivo Scotchbond MP (3M/ESPE - St Paul, MN, EUA) e, posteriormente, foi fixado um fio ortodôntico com resina composta 
Z250 (3M/ESPE - St Paul, MN, EUA). O tracionamento foi realizado por amarrias, as quais foram substituídas semanalmente, durante um mês. Obteve-se extrusão dental de aproximadamente $2 \mathrm{~mm}$, sendo suficiente para que a linha de término da fratura se apresentasse acima do limite gengival, permitindo, assim, a futura colagem do fragmento (Figura 4).

Figura 4 - Tracionamento dental inicial

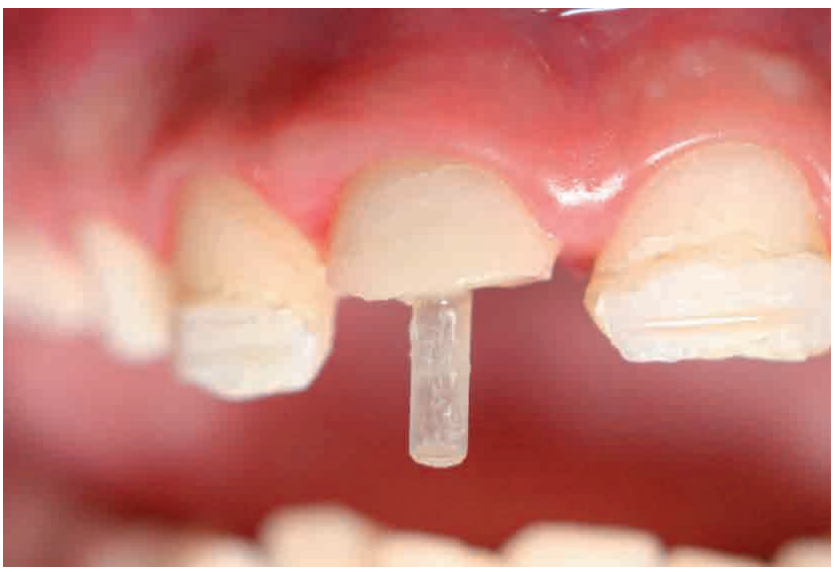

Após esse período, o aparelho foi mantido em posição por 8 semanas, para estabilização do periodonto. Realizou-se a remoção do fio ortodôntico no interior do canal.

Para a realização da colagem do fragmento dental, foi realizada nova profilaxia dos dentes ântero-superiores e preparado conduto radicular para cimentação do pino de fibra de vidro White Post DC (FGM, Joinville, Brasil). A remoção do material obturador e o desgaste das paredes internas do canal foram realizados com as brocas do sistema de pinos compatível ao diâmetro do canal.

Como preparo do remanescente para a colagem, foi realizado o condicionamento ácido e posterior lavagem e secagem com cones de papel absorvente. Em seguida, foi aplicado o sistema adesivo Scothbond MP Plus com associação do Ativator e Catalizador no interior do canal radicular (3M/ESPE, St. Paul, MN, EUA). Anteriormente ao procedimento de cimentação, o pino de fibra de vidro foi silanizado com o silano Angelus (Angelus, Londrina, Brasil).

Após todo procedimento de tratamento do remanescente radicular e do pino pré-fabricado, foi realizada a cimentação com cimento resinoso Rely X ARC (3M - ESPE St Paul, MN, USA). O cimento foi espatulado em placa de vidro por 15 segundos e inserido no conduto radicular, com auxílio de uma broca lentulo, e no pino pré-fabricado. A fotopolimerização foi realizada por 5 segundos e, logo após, o excesso de cimento resinoso foi removido.

Para o tratamento do fragmento dental, foi realizado o procedimento tradicional de aplicação do sistema adesivo. Em seguida, o fragmento foi levado em posição (Figura 5) e polimerizado por 20 segundos. Para preencher os espaços entre o remanescente dental e o fragmento, utilizou-se a resina composta Z250 (3M/ESPE, St. Paul, MN, EUA) em incrementos de 1 $\mathrm{mm}$ fotopolimerizados durante 20 segundos (Figura 6).

Figura 5 - Adaptação do fragmento

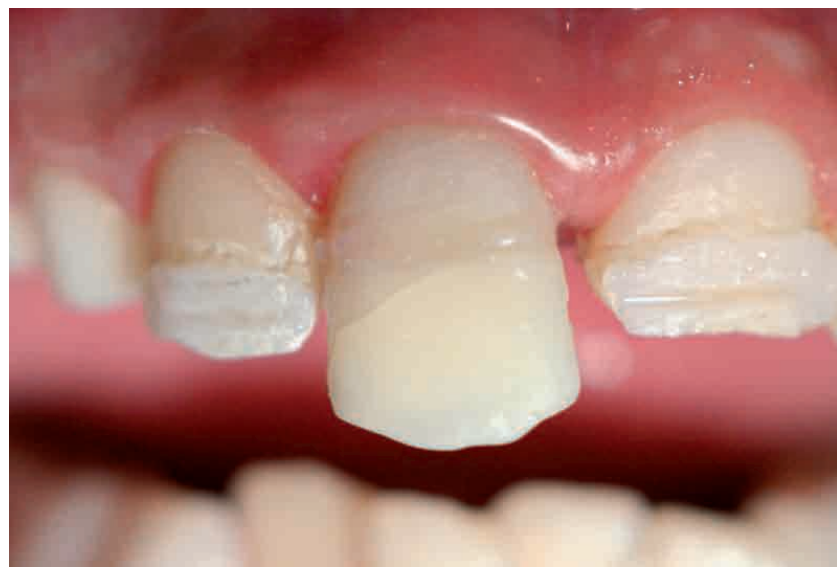

Figura 6 - Restauração do elemento dental 11

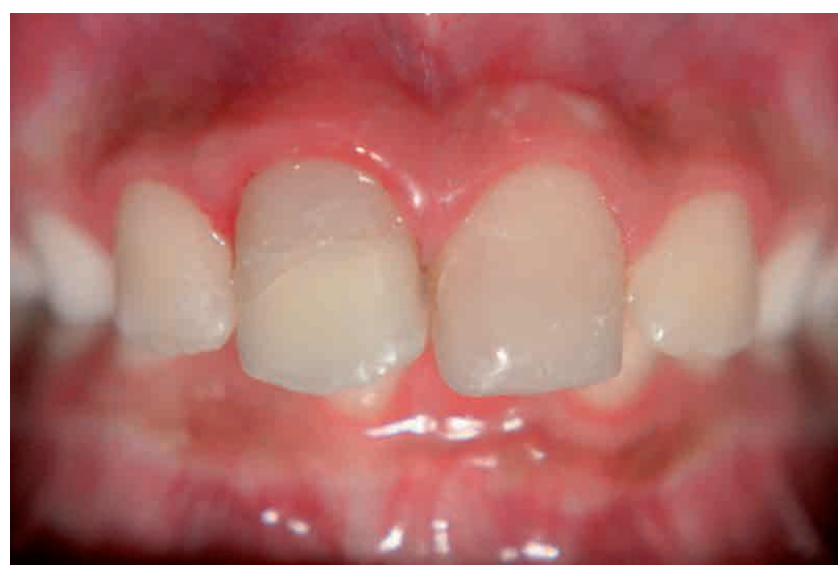

O polimento final foi realizado na mesma sessão utilizando pontas de silicone Enhance (Dentsply, York, PA, EUA) e discos de lixa Sof-Lex (3M/ESPE, St. Paul, MN, EUA).

Após o procedimento concluído, foram realizadas radiografias para confirmar o assentamento do pino pré-fabricado, e observou-se, ainda, a fina linha 
de cimento obtida entre o pino anatômico e a parede do canal e um íntimo contato entre pino e dente.

Ao término do tratamento, constatou-se satisfação por parte do paciente e de seus responsáveis, principalmente no que tange à estética obtida. Os responsáveis pelo paciente foram orientados a procurar a clínica odontológica da PUC/PR em 6 meses, ou antes desse prazo, caso alguma anormalidade ocorresse. Entretanto, não houve retorno após esse período, nem mesmo o contato via telefone foi conseguido.

\section{DISCUSSÃO}

São várias as técnicas de tratamento sugeridas para casos de fratura de dentes anteriores traumatizados, dependendo a escolha da situação clínica (MILARDOVIC ORTOLAN et al., 2012). Seja qual for a técnica escolhida, a distância biológica entre a base do sulco gengival histológico e a crista óssea deve ser respeitada, sob pena de inflamação gengival, perda de inserção e consequente formação de bolsa periodontal (SANTOS; SARTORI, 2000).

Seguindo as conclusões da pesquisa de Gargiulo et al., em 1961, buscou-se, neste caso, obter $3 \mathrm{~mm}$ entre a base do sulco gengival e a crista óssea; espaço necessário para acomodar o epitélio juncional, inserção das fibras gengivais e sulco gengival. A literatura sugere que, desde que haja quantidade de gengiva suficiente e se a linha de fratura se localizar no máximo $1 \mathrm{~mm}$ abaixo da margem gengival, pode-se optar pelo tracionamento ortodôntico (DURHAM; GODDARD; MORRISON, 2004). Essa técnica de tratamento pode ser dividida em rápida e lenta. No tracionamento rápido, são usadas forças constantes acima de $60 \mathrm{~g}$ com ativação em pequenos intervalos (3 a 5 dias) (LEMON, 1982). Essa combinação de grande força de ativação/ pequeno intervalo de ativação provoca apenas exposição da raiz sem acompanhamento do tecido gengival (LEMON, 1982; JANSON et al., 2002; NORMANDO et al., 2004). No tracionamento lento, são usadas forças de 20 a 30 g, com ativações de 13 a 15 dias, proporcionando uma movimentação do tecido gengival juntamente à raiz dentária (JANSON et al., 2002). Assim, a escolha dessa modalidade de tratamento para recuperação das distâncias biológicas justifica-se, pois trata-se de um dente anterior, em que um desnível gengival poderia causar um comprometimento estético. Além disso, a literatura também cita a facilidade de realização e o excelente prognóstico como vantagens da extrusão ortodôntica (BACH et al., 2004; BAJAJ et al., 2015). O tempo de contenção usado no caso (8 semanas) foi respaldado pela literatura, que recomenda de 8 a 12 semanas para esse fim (BAJAJ et al., 2015).

A técnica da colagem do fragmento dental apresenta várias vantagens sobre a realização de uma restauração direta de resina composta: simplificação técnica, menor tempo clínico, restabelecimento imediato da estética e da função, menor desgaste familiar, preservação de guias funcionais, estabilidade de cor e melhor adaptação incisal (SHARMA et al., 2011; MAZZOLENI et al., 2016).

A necessidade da intervenção endodôntica a longo prazo em pacientes jovens acometidos de traumatismos dentais é muitas vezes necessária, devido às características histológicas do complexo dentinho-pulpar (ANDRADE et al., 1999). Além disso, devido à integridade estrutural estar reduzida causando o enfraquecimento do dente, optou-se pelo tratamento endodôntico para que, juntamente a um procedimento restaurador, fosse oferecida proteção ao remanescente dentário (MAZZOLENI et al., 2016). A realização da gengivoplastia foi motivada pela necessidade de melhores condições para aposição do isolamento absoluto. Essa técnica cirúrgica é semelhante à gengivectomia, diferenciando-se desta pelo objetivo de se conseguir um recontorno gengival fisiológico (NASSAR; BITENCOURT; NASSAR, 2011). O tempo de cicatrização dos tecidos gengivais após a gengivoplastia é, inclusive, o mesmo de uma gengivectomia: 14 dias (DEAS et al., 2016).

Nesse caso, optou-se pelo pino de fibra de vidro, o qual apresenta como vantagens: baixo stress, módulo de elasticidade semelhante ao da dentina, cor translúcida, favorecendo a estética; fácil remoção com instrumentos rotatórios, custo menor do que outros sistemas, ausência de corrosão, compatibilidade com os sistemas adesivos e cimentos resinosos, formato que proporciona alta retentividade mecânica e menor desgaste da estrutura dentária próximo ao ápice (FERRARI; VICCHI, 2000; CECCHIN et al., 2016).

A cimentação adesiva tem ganhado muita popularidade devido à capacidade de formar uma união efetiva com a dentina e reforçar a estrutura radicular fragilizada. Contudo, a resistência de união de cimento resinoso e pino de fibra é um fator crítico para o sucesso de procedimentos endodônticos (SILVA et al., 2015). A utilização do pino foi associada à colagem 
do fragmento, favorecendo um alicerce seguro sobre o qual foi confeccionada a restauração.

\section{CONCLUSÃO}

Os resultados obtidos permitem concluir que a associação de diferentes áreas da odontologia, interagindo juntas, pode apresentar resultados satisfatórios para o restabelecimento estético e funcional dos dentes anteriores, desde que corretamente indicada e executada.

A reabilitação de dentes com grandes porções de estrutura dentária destruída e/ou com raízes fragilizadas necessitam de uma retenção intrarradicular que se adapte bem à morfologia do conduto.

O paciente deve estar informado de todas as possibilidades de tratamento e dos riscos inerentes. O cirurgião-dentista deve estar atualizado dentro dos diferentes procedimentos clínicos a fim de selecionar o tratamento mais adequado para cada situação clínica.

\section{REFERÊNCIAS}

ANDERSSON, L. Epidemiology of traumatic dental injuries. Journal of Endodontics. v.39, suppl. 3, p. S2-5, 2013.

ANDRADE, W. B. et al. Repercussões de traumatismo dental recorrente durante o tratamento de pacientes já traumatizados. ECLER Endodontics. v. 1, n. 3, 1999.

ANDREASEN, J. O. Fundamentos de traumatismo dental. $2^{\mathrm{a}}$ ed Porto, Alegre: Artmed, 2001.

ANDREASEN, J. O.; LAURIDSEN, E.; ANDREASEN, F, M. Contradictions in the treatment of traumatic dental injuries and ways to proceed in dental trauma research. Dental Traumatology. v.26, p. 16-22, 2010.

BACH, N.; BAYLARD, J. F.; VOYER, R. Orthodontic extrusion: Periodontal considerations and applications. Journal Canadian Dental Association. v. 70, p. 775-780, 2004.

BAJAJ, P. et al. Multidisciplinary approach to the management of complicated crown-root fracture: a case report. Journal International Oral Health. v. 7, n. 4, p. 88-91, 2015.

CECCHIN, D. et al. Acid Etching and Surface Coating of Glass-Fiber Posts: Bond Strength and Interface Analysis. Brazilian Dental Journal. v. 27, n. 2, p. 228-233, 2016.

DEAS, D. E. et al. Scaling and root planing vs. conservative surgery in the treatment of chronic periodontitis. Periodontology 2000. v. 71, n. 1, p. 128-39, 2016.
DURHAM, T. M.; GODDARD, T.; MORRISON, S. Rapid forced eruption: a case report and review of forced eruption techniques. General Dentistry. v. 52, n. 2, p.167-175, 2004.

FERRARI, M.; VICCHI, A. A retrospective study of fiberreinforced epoxy resin posts versus cast posts and cores: a four year recall. American Journal of Dentistry. v. 13, p. 9B-14B, 2000.

FERRARI, M.; VICCHI, A. A retrospective study of fiberreinforced epoxy resin posts versus cast posts and cores: a four year recall. American Journal of Dentistry. v. 13, p. 9B-14B, 2000.

GÓES, K. K. H. Avaliando os traumatismos dentoalveolares: revisão de literatura. Revista de Cirurgia e Traumatologia Buco-Maxilo-Facial. v. 5, n.1, p.21-26, 2005.

GÓES, K. K. H. et al. Avaliando os traumatismos dentoalveolares: revisão de literatura. Revista de Cirurgia e Traumatologia Buco-Maxilo-Facial. v. 5, n.1, p. 21-26, 2005.

GONG, Y. et al. Emergency dental injuries presented at the Beijing Stomatological Hospital in China. Dental Traumatology. v. 27, p. 203-207, 2011.

JANSON, M. R. P. et al. Tratamento interdisciplinar II Estética e distância biológica: alternativas ortodônticas para remodelamento vertical do periodonto. Revista Dental Press de Ortodontia e Ortopedia Facial. v. 7, n. 4, p. 85105, 2002.

LEMON, R. R. Simplified esthetic root extrusion techniques. Oral Surgery, Oral Medicine, Oral Pathology. v. 54, n. 1, p. 93-9, 1982.

LEVIN, L. et al. Dental and oral trauma during childhood and adolescence in Israel: occurrence, causes, and outcomes. Dental Traumatology. v.23, p. 356-359, 2007.

LO GIUDICE, G. et al. Tooth fragment reattachment technique on a pluri traumatized tooth. Journal of Conservative Dentistry. v. 15, p. 80-83, 2012.

MANJU, M.; SRINIVAS, L. S.; SAVITHA, K. C. Esthetic and biologic mode of reattaching incisor fracture fragment utilizing glass fiber post. Journal of Natural Science, Biology and Medicine. v. 6, n. 2, p. 446-448, 2015.

MAZZOLENI, S. et al. Influence of Root Canal Posts on the Reattachment of Fragments to Endodontically Treated Fractured Incisors: An in vitro Experimental Comparison. Journal of Esthetic and Restorative Dental. v. 28, n.2, p. 92-101, 2016.

MILARDOVIC ORTOLAN, S. et al. Esthetic rehabilitation of complicated crown fractures utilizing rapid orthodontic extrusion and two different restoration modalities. International Journal of Clinical Pediatric Dentistry. v. 5, n. 1, p. 64-67, 2012. 
MORAES, M. de et al. Estudo comparativo entre procedimento de colagem do botão ortodôntico para tracionamento de dentes retidos. Revista Dental Press de Ortodondia e Ortopedia Facial. Maringá, v. 3, n. 5, p. 5258, 2008.

NORMANDO, A. D. C. Extrusão Ortodôntica como recurso no tratamento das invasões dos espaços biológicos periodontais - Indicação clínica e divulgação de um método simplificado de tratamento. Jornal Brasileiro de Ortodontia e Ortopedia Facial. v. 9, p. 53, p. 502-10, 2004.

PETERSON, L. J. et al. Cirurgia oral e maxilofacial contemporânea. $3^{\mathrm{a}}$. ed. Rio de Janeiro: Guanabara Koogan, 2000 .

QUINTAS, A. F.; DINATO, J. C.; BOTTINO, M. A. Aesthetic posts and cores for metal-free restoration of endodontically treated teeth. Practice Periodontics Aesthetic Dentistry Journal. v. 12, n. 9, p. 875-884, 2000.

SANTOS, F. A.; SARTORI, R. Cirurgia Periodontal para o Restabelecimento das Distâncias Biológicas: Relato de um Caso Clínico. Publicatio UEPG - Biological and Health Sciences. v. 6, n. 1, p. 89-101, 2000.

SHARMA, D. et al. Multidisciplinary approach to the rehabilitation of a tooth with two trauma episodes: systematic review and report of a case. Dental Traumatology. v. 27, n. 4, p. 321-326, 2011.

SILVA NR et al. Effect of Resin Cement Porosity on Retention of Glass-Fiber Posts to Root Dentin: An Experimental and Finite Element Analysis. Brazilian Dental Journal. v.26, n. 6, 2015.

YOUSEF, M. K. Reattachment of fractured teeth fragments in mandibular incisors: a case report. Internation Medical Case Reports Journal. v. 8, p. 87-91, 2015. 